Research Article

\title{
Theoretical Study on Effect of Defective Connection to Reservoirs in an Atomic-Scale Conductor
}

\author{
Masato Morifuji \\ Department of Quantum Electronics, Graduate School of Engineering, Osaka University, Suita, Osaka 565-0871, Japan \\ Correspondence should be addressed to Masato Morifuji; morifuji@eei.eng.osaka-u.ac.jp
}

Received 21 March 2017; Revised 7 July 2017; Accepted 20 July 2017; Published 21 August 2017

Academic Editor: Sergio E. Ulloa

Copyright (C) 2017 Masato Morifuji. This is an open access article distributed under the Creative Commons Attribution License, which permits unrestricted use, distribution, and reproduction in any medium, provided the original work is properly cited.

We theoretically investigate the effect of a defect at the interface between a conductor and reservoirs in an atomic-scale device. Since fabrication of atomic-scale contacts is a complex task, there could be defects at the interface between the conductor and reservoirs. Such defective contacts will make it difficult to measure currents properly. In this paper, we calculate current-voltage characteristics in two-dimensional devices with a defective connection to reservoirs by using the nonequilibrium Green's function method. Results show that the magnitude of resistance change depends on the amplitude of quantized wave functions at the position of the defect.

\section{Introduction}

Atomic-scale conductors such as nanowires and nanoribbons are attracting much attention. There are many studies motivated by a prospect that smaller devices would show superior performance: higher speed, higher sensitivity, and less power consumption. Not only carbon (graphene) [1,2] but also various materials such as $\mathrm{ZnO}$ [3], BiSe [4], GaN [5], Si [6], and $\mathrm{Fe}$ [7] are used for fabricating nanoribbons. The large variation of the purpose of the studies, such as a single atom transistor [8-11], a laser [3], a thermal device [4], a magnetic sensor [2], and skin prosthesis [6], indicates the high potential of the atomic-scale devices. In addition to these conventional devices, there are investigations of exotic phenomena such as spin-polarized current [7] and coherent many-body current [12].

In exploiting transport phenomena in the atomic-scale devices, connection to reservoirs is indispensable. Since fabrication of such a small contact is a complex task, defects will be easily introduced at the interface between the conductor and reservoirs. Even if characteristics of the conductor are interesting and useful, improper connection to reservoirs will rectify the current and will smear out the features that we want to observe. Therefore, connection to reservoirs is of special importance for transport measurements in atomicscale devices. The effect of a defect at the interface, however, has not been well understood.
In this paper, by using the nonequilibrium Green's function method [13], we theoretically investigate the effect of the defect at the connection to reservoirs in two-dimensional atomic-scale conductors within a simple model for the defect.

\section{Model Structure}

Figure 1 shows a schematic structure of the device we investigate. We calculate current-voltage characteristics of two-dimensional conductors with a ribbon-like shape. We simulate the device by discretizing it into pieces of the size $\Delta x \times \Delta x$. We call the piece a virtual atom. The device is expressed as an array of the atoms as shown by the dots in Figure 1. As we show in the upper part of this figure, the conductor consists of an $N_{d} \times N_{w}$ array of the virtual atoms. There are reservoirs (anode and cathode) attached on both sides of the conductor as indicated by the shaded areas. We set the size of the reservoirs large enough so that energy spacing between electronic levels therein is smaller than the thermal energy $k_{B} T$. As shown in the lower part, there is diffusion potential $\Delta$ between the conductor and reservoirs. The reservoirs are filled with electrons up to the Fermi energy $E_{F}$.

Considering that each atom is associated with a single s-like orbital, we introduce a hopping interaction between neighboring atoms denoted by " $-t$." We describe the defect as 


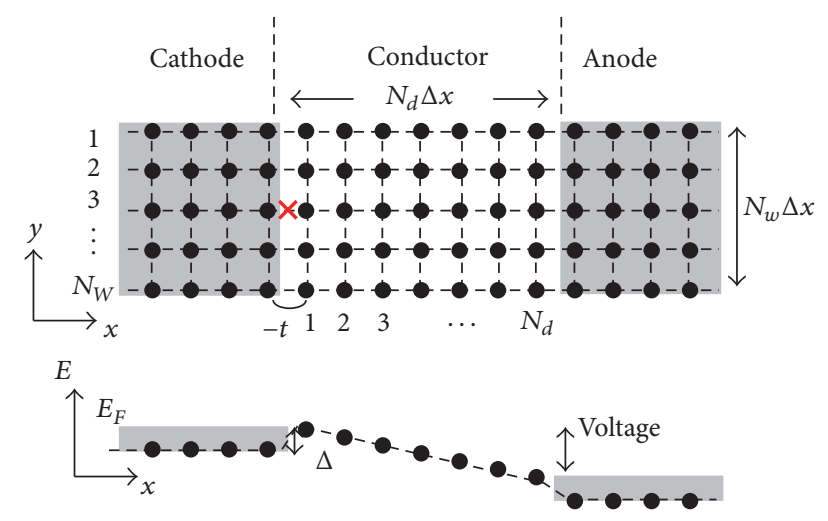

Figure 1: Model of the device studied in this paper. Virtual atoms, denoted by dots, are associated with an s-like orbital, and neighboring orbitals are connected by hopping interaction " $-t$." The conductor consists of an $N_{d} \times N_{w}$ array of the virtual atoms. On both sides of the conductor, there are reservoirs shown by shaded areas. A defect between reservoirs and conductor indicated by cross where the hopping interaction is replaced by a smaller value.

disconnection of the hopping interaction; that is, we replace $t$ by $\tau(0 \leq \tau / t<1)$ on the defective bond as indicated by the cross in Figure 1. Although this model is very simple to simulate realistic defects, we believe that this model contains the essential features of the function of the defect.

In the present calculations, we set the spacing between the virtual atoms to $\Delta x=0.5 \mathrm{~nm}$. We also set the diffusion potential between reservoirs and conductor to $\Delta=0.1 \mathrm{eV}$ and the Fermi energy in the reservoirs to $E_{F}=0.1 \mathrm{eV}$. The magnitude of the hopping interaction is determined as follows: the hopping interaction yields the energy band $\epsilon_{k}=$ $-2 t \cos (k \Delta x)$, where $k$ is a wave vector. For small $k$, we have $\epsilon_{k} \sim t k^{2} \Delta x^{2}$. Equalizing this expression to $\hbar^{2} k^{2} / 2 m^{*}$, we have a relation between $t$ and the effective mass $m^{*}$ as $t=$ $\hbar^{2} /\left(2 m^{*} \Delta x^{2}\right)$. For example, $m^{*}=0.1 m_{0}\left(m_{0}\right.$ is the electron rest mass) corresponds to the hopping interaction $t=1.51 \mathrm{eV}$.

We note that we consider a square array of the atoms as shown in Figure 1. Graphene attracts much interest for its peculiar band structure arising from the honeycomb atomic array. To investigate the genuine effect of the defects, however, we adopted a device structure that shows normal conduction.

\section{Nonequilibrium Green's Function Theory}

Green's function is a matrix defined by an equation:

$$
[E \mathbf{1}-H] G(E)=\mathbf{1},
$$

where $H$ is a Hamiltonian matrix for the whole system and 1 denotes a unit matrix of the corresponding dimension. The Hamiltonian consists of the hopping interaction $t$ and potential energies for each of the atoms. By dividing the whole system into three parts, the left reservoir, the device, and the right reservoir, we can derive the equation

$$
\left[E \mathbf{1}-H^{d}-\Sigma^{L}(E)-\Sigma^{R}(E)\right] G^{d}(E)=\mathbf{1},
$$

where $H^{d}$ and $G^{d}$ are the Hamiltonian and Green's function of the device. The quantity $\Sigma^{L}\left(\Sigma^{R}\right)$, called self-energy, denotes a strength of the connection between the reservoir and the device. Details of the theory and formulations are written in $[13,14]$. Solving (2), we obtain $G^{d}(E)$, from which charge density is evaluated by the equation

$$
\begin{aligned}
\rho & =\frac{2}{\pi} \\
& \cdot \int_{0}^{\infty} d E \operatorname{Tr}\left[G^{d}\left(\operatorname{Im} \Sigma^{L} f_{L}(E)-\operatorname{Im} \Sigma^{R} f_{R}(E)\right) G^{d}\right],
\end{aligned}
$$

where $f_{L}(E)\left(f_{R}(E)\right)$ is the Fermi-Dirac distribution for the left (right) reservoir. From the charge density, Coulomb interaction between electrons is evaluated. The Coulomb interaction determines the Hamiltonian from which Green's function is calculated. We repeat this self-consistent procedure until we obtain a stable solution. Once we have the stable solution, we can calculate the current-voltage characteristics with the equation

$$
I(V)=\frac{e}{\pi \hbar} \sum_{j, j^{\prime}=1}^{N_{W}} \int_{0}^{\infty} d E\left[I_{R \rightarrow L}(E)-I_{L \rightarrow R}(E)\right],
$$

where $I_{L \rightarrow R}(E)$ denotes electron flow from the left reservoir (cathode) to the right reservoir (anode). The quantity $I_{L \rightarrow R}(E)$ is written as

$$
\begin{aligned}
I_{L \rightarrow R}(E)= & 4\left|G_{\left(1, j ; N d, j^{\prime}\right)}^{d}(E)\right|^{2}\left(f_{L}(E) \operatorname{Im} \Sigma_{(1, j ; 1, j)}^{L}\right) \\
& \cdot\left[\left(1-f_{R}(E)\right) \operatorname{Im} \Sigma_{\left(N d, j^{\prime} ; N d, j^{\prime}\right)}^{R}\right] .
\end{aligned}
$$

In (5), the element of Green's function $G_{\left(1, j ; N d, j^{\prime}\right)}^{d}(E)$ denotes amplitude that an electron located at position $(1, j)$ of the device arrives at position $\left(N_{d}, j^{\prime}\right)$. The second (the third) factor indicates the strength of the connection between the cathode (anode) and the device. Similarly, the electron flow from the anode to the cathode is given by

$$
\begin{aligned}
I_{R \rightarrow L}(E)= & 4\left|G_{\left(1, j ; N d, j^{\prime}\right)}^{d}(E)\right|^{2} \\
& \cdot\left[\left(1-f_{L}(E)\right) \operatorname{Im} \Sigma_{(1, j ; 1, j)}^{L}\right] \\
& \cdot\left(f_{R}(E) \operatorname{Im} \Sigma_{\left(N d, j^{\prime} ; N d, j^{\prime}\right)}^{R}\right) .
\end{aligned}
$$

\section{Results and Discussion}

In Figure 2, we plot the current-voltage curves for the structures containing a single defect between the cathode and conductor as illustrated in the inset (right down). In all the calculations, we set the temperature $T=300 \mathrm{~K}$. Although there are a large number of variations of defect configurations, we treat here only a single-point defect. These curves were obtained for the conductor size $N_{d}=20$ and $N_{w}=10$ and the effective mass $m^{*}=0.1 m_{0}$. We set the hopping interaction on the defect bond as $\tau=0$. The curve labeled "No defect" shows a current when there are 


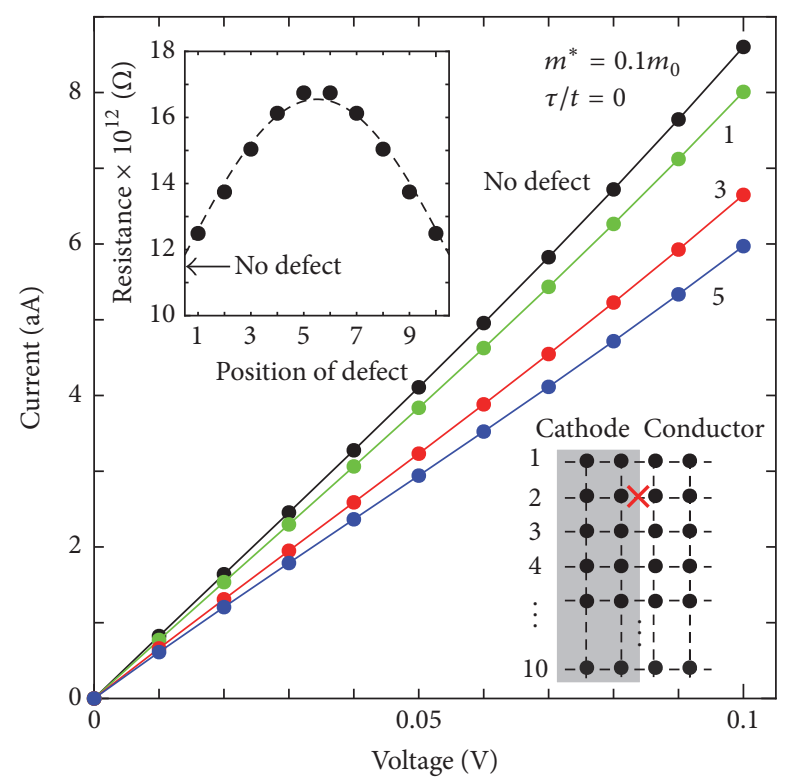

FIgURE 2: Current-voltage characteristics calculated for size of the conductor $N_{d}=20$ and $N_{w}=10$ and effective mass $m^{*}=0.1 m_{0}$. The right inset shows schematic structure at the cathode-conductor interface where cross denotes the defect. The curves labeled " 1, , " 3 ," and " 5 " denote current for a defect at the 1 st, $3 \mathrm{rd}$, and 5 th rows in the interface, respectively. The left inset shows the resistance as a function of the defect position.

no defects at the interface. The curves labeled "1," " 3 ," and "5" show currents calculated for the structure containing a single defect at the first, third, and fifth rows, respectively. We note that the defect at " 1, " " 3 ," and " 5 " positions gives rise to the same results as the defect at " 10 ," " 8 ," and " 6 " positions, respectively, because of symmetry in the lateral ( $y$ ) direction. Defect at the interface between the conductor and anode yields similar results as long as the voltage drop across the conductor is less than the spacing of energy levels in the conductor, although the results are not shown.

The defect gives rise to decrease in current, that is, increase in resistance. The resistance increase occurs simply because a defect prevents electrons from flowing through the device. A defect located in the middle of the device gives rise to a larger increase in resistance than that near the edge of the device. In the inset (left up) of Figure 2, we plot resistance $R_{n}$ defined by

$$
R_{n}=\left.\frac{\partial V}{\partial I}\right|_{V=0}
$$

for the structure with a defect at the $n$th position as a function of the position of the defect $n$. Resistance for the structure without defect is indicated by the horizontal arrow. The behavior of the resistance is well given by a sine function as indicated by the dashed curve.

In Figure 3, we show the current-voltage relation for $\tau=0 \sim 1$ in the fifth position. The current decreases with decreasing $\tau$. This means that we obtain the similar results for

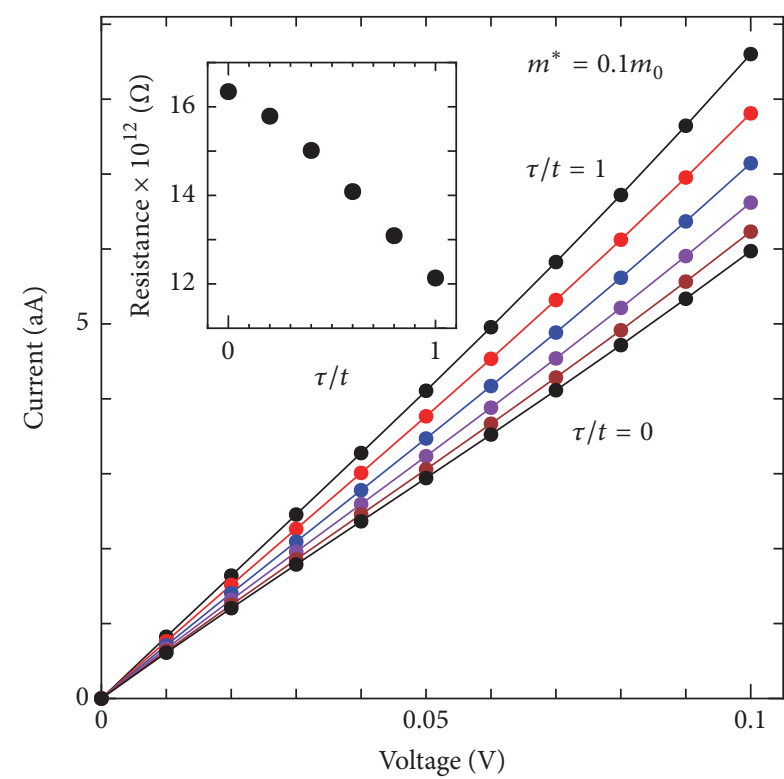

FIgURE 3: Current-voltage characteristics calculated for $\tau / t=0.0$, $0.2,0.4,0.6,0.8$, and 1.0. The size of conductor is $N_{d}=20$ and $N_{w}=$ 10 and effective mass $m^{*}=0.1 m_{0}$. The inset shows the resistance as a function of $\tau / t$.

$\tau=0$ even when $\tau \neq 0$; although the magnitude is different, the resistance shows sinusoidal position dependence.

Considering that discrete energy levels are formed due to confinement of electron in the lateral direction, we understand the defect position dependence of the resistance. Since each of the discrete levels is associated with a sinusoidal wave function, we can attribute the behavior of resistance against defect position to the amplitude of the quantized lateral wave functions. Current injection from the cathode into the conductor is prevented by the defect. The strength of the current flowing from the cathode into the conductor depends on the amplitude of the wave function in the conductor. The amplitude of the lateral wave function is small at the edge of the device. Therefore, the effect of the defect at the edge is small. On the contrary, when the defect locates at the middle of the interface, current injection is largely affected, and resistance change is also large.

To ascertain the role of the wave functions of the lateral modes, we calculated charge densities in the conductor as shown in Figure 4(a). These figures show charge densities for the structure without defect and with a defect at the first $(n=1)$, the third $(n=3)$, and the fifth $(n=5)$ positions as indicated by the cross in each figure. The charge density near the defect position is reduced.

In Figure 4(b), we plot the charge density along the interface between the cathode and conductor, that is, along with $y$-axis at $x=1$. The charge density adjacent to the defect is indicated by the big symbol for each curve. Note that the curves are results of the spline fitting guiding for eyes. Reduction of charge density occurs for any value of $n$. However, the curve labeled $n=5$ indicates that large reduction in charge density occurs when the defect locates 

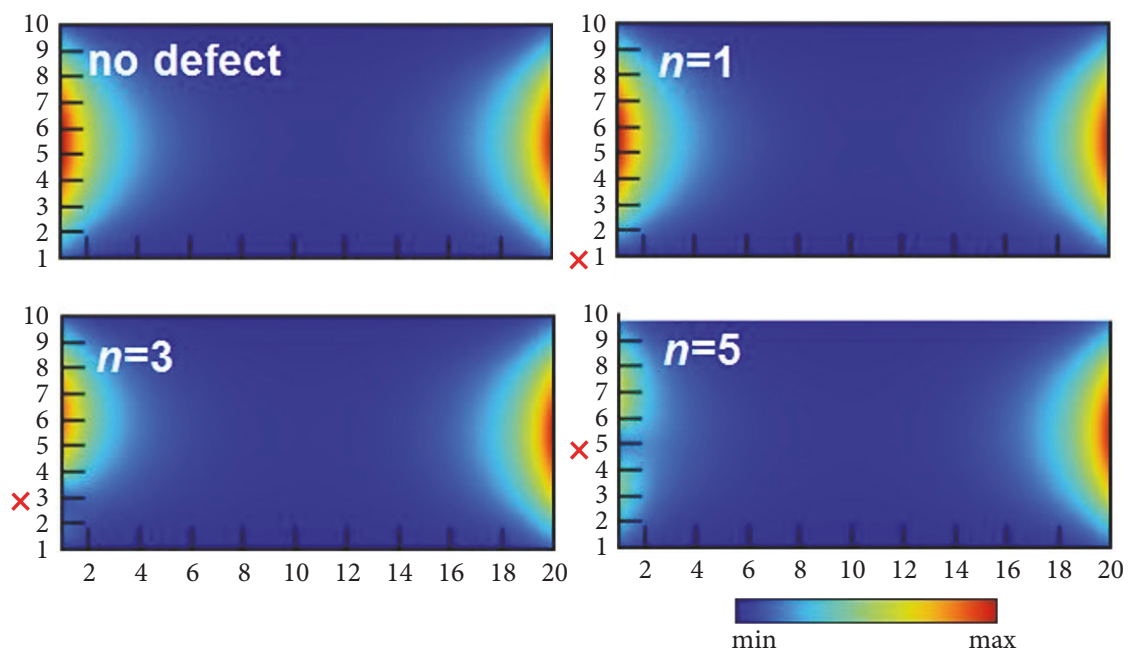

(a)

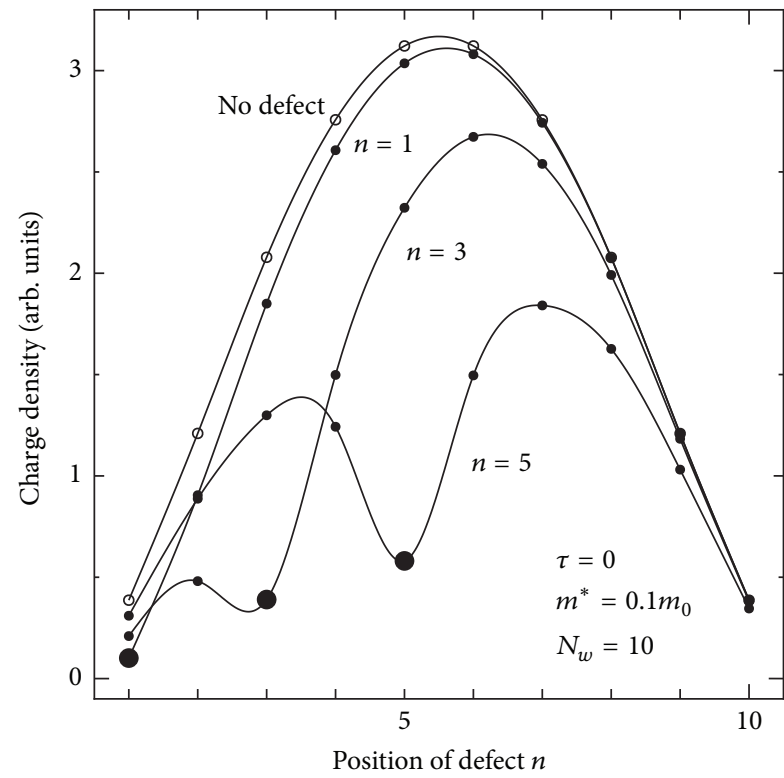

(b)

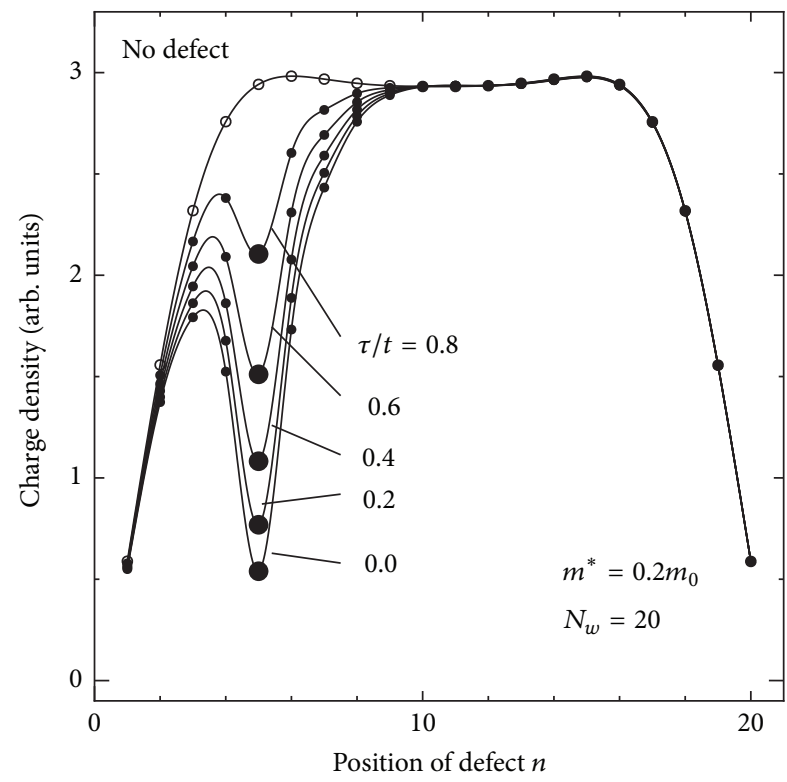

(c)

FIGURE 4: (a) Charge densities calculated for structures without defect and with a defect at the first, third, and fifth positions, respectively. (b) Charge densities along the interface between the cathode and conductor. The large dots indicate the position adjacent to the defect. In (a) and (b), we set $m^{*}=0.1 m_{0}, N_{W}=10$, and $\tau=0$. (c) Charge densities along the interface between the cathode and conductor for $m^{*}=0.2 m_{0}$ and $N_{W}=20$ and various values of $\tau$ with the defect at the fifth site.

in the middle of the device where the quantized lateral mode has large amplitude. In Figure 4(c), we also show the charge density for the structure $m^{*}=0.2 m_{0}, N_{W}=20$, and the defect at the fifth site. The curves are for the values of $\tau$ between 0 and 1 . As already shown in Figure 3, results for $\tau \neq 0$ are qualitatively the same as those for $\tau=0$. In what follows, thus, we show the results evaluated for $\tau=0$.

To examine further the role of the quantized lateral wave functions, we carried out calculations for wider devices. We show the results in Figure 5. In this figure, we plot $\Delta R / R_{0} \equiv$ $\left(R_{n}-R_{0}\right) / R_{0}$, that is, change of resistance normalized by the resistance without the defect $R_{0}$ as a function of defect position $n$ for various values of $N_{w}$. Note that the curves are offset, and open circles in each curve denote the value of $R_{0}$ for each structure. The curve for $N_{w}=10$ is the same as the curve shown in the inset of Figure 2. As we noted, this curve is well fitted by a sine curve. With increasing $N_{w}$, the curve becomes flat with a plateau in the intermediate values of $n$, showing that position dependence of resistance change becomes smaller with device width. With further increase of $N_{w}$, the curve shows a shallow dip at about $n \simeq N_{w} / 2$.

Considering that a wave function confined in $y$-direction within a region $W$ is given by

$$
\psi_{n_{y}}(y)=\sqrt{\frac{2}{W}} \sin \left(\frac{n_{y} \pi y}{W}\right)
$$




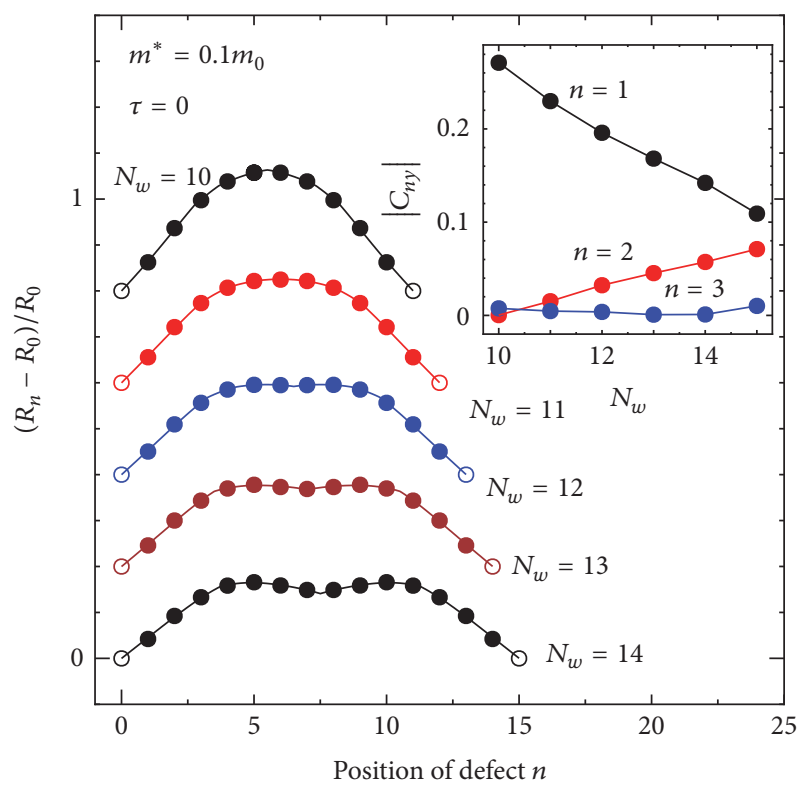

FIGURE 5: Change in resistance $\left(R_{n}-R_{0}\right) / R_{0}$ for $m^{*}=0.1 m_{0}$ is plotted by dots as a function of defect position $n$ for the width $N_{w}=$ $10 \sim 14$. Curves are results of fitting to (9). Inset: coefficient $\left|C_{n}\right|$ in (9) evaluated by the least square fitting.

with the quantum number $n_{y}=1,2,3, \ldots$, we fitted the values of $\Delta R / R_{0}$ with the function

$$
\frac{\Delta R(y)}{R_{0}}=\sum_{n_{y}=1}^{3} C_{n_{y}}\left|\sin \left(\frac{n_{y} \pi y}{\left(N_{w}+1\right) \Delta x}\right)\right| .
$$

We show the fitted results by solid curves in Figure 5 . Good agreement has been obtained by the superposition of quantized lateral wave functions as given by (9). In the inset of Figure 5, we plot the value of $\left|C_{n_{y}}\right|$ evaluated by the fitting. For smaller values of $N_{w}, C_{1}$ is much larger than others. This result indicates that the ground state lateral mode, that is, wave function with the quantum number $n_{y}=1$, is dominating current. With increasing $N_{w}$, a ratio of lateral mode with $n_{y}=2$ becomes larger. This is because energy of quantized state becomes lower with increasing device width. The shallow dip seen in the curves for $N_{w}=13$ and 14 is a result of the participation of the $n_{y}=2$ state that has a node at $N_{w} / 2$.

The enrolment of the second state appears more clearly when the electron effective mass is larger because the higher levels lie in lower energies. Figure 6 shows the calculated results for the effective mass $m^{*}=0.2 m_{0}$. The curves of $\Delta R_{n} / R_{0}$ indicate that resistance change is small when the defect is located in the middle of the device. In other words, the defect that locates in the middle of the device does not prevent electrons from flowing into the conductor. This result indicates that the second state that has a node in the middle of the device is dominant in current flow. As shown in the inset, component of the $n_{y}=2$ state, which has a node in the middle of the device, is larger than others.

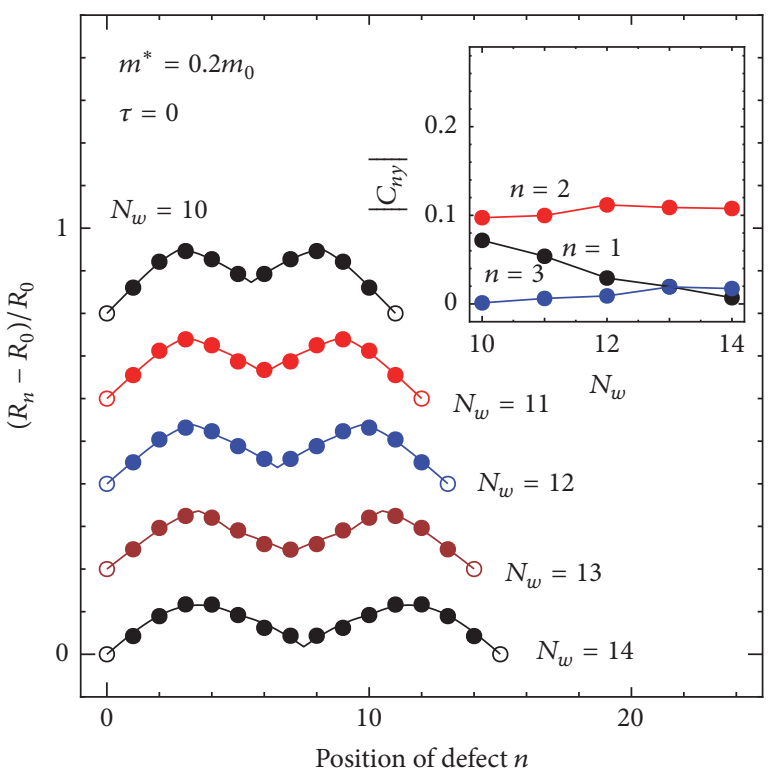

FiguRE 6: Change in resistance $\left(R_{n}-R_{0}\right) / R_{0}$ for $m^{*}=0.2 m_{0}$.

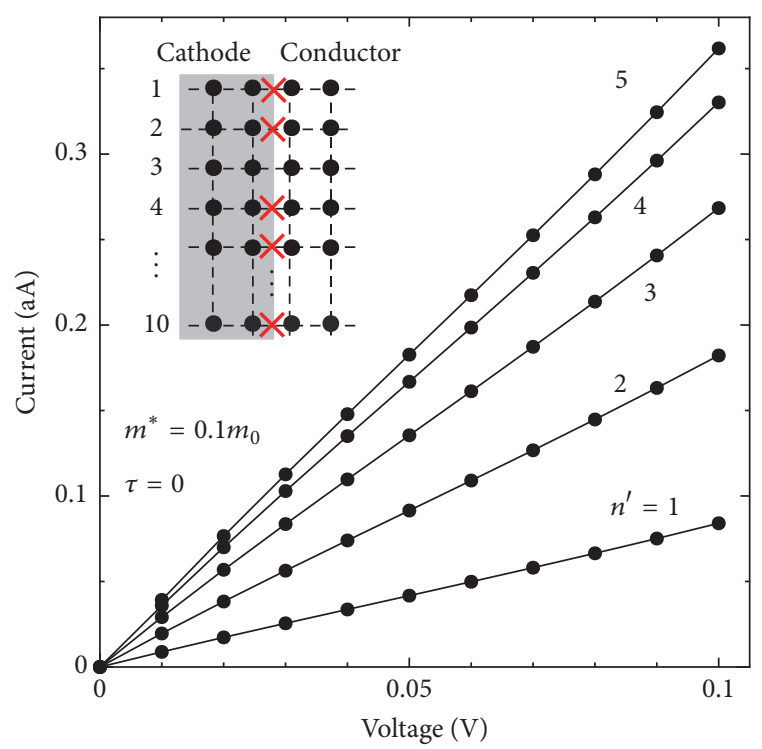

FIGURE 7: Current-voltage characteristics for structures containing defects except for a single point as schematically shown in the inset.

The defect position dependence of the resistance described so far might be difficult to be observed because of difficulty in preparing samples containing position-controlled defects. We would realize similar situations by using the scanning tunneling microscopy (STM) technique. In Figure 7, we show $I-V$ curves for structures with a singlepoint connection; that is, bonds except the $\bar{n}$ th position at the interface are disconnected as schematically shown in the inset. In this case, $R_{\bar{n}}^{-1}$ shows sine-like behavior because a connection in the middle of the device enlarges current (i.e., reduces resistance) more than a connection near the edge of the device does. Such a situation of a single-point connection 
to reservoir would be realized by using an STM tip as a cathode or anode. Since only the topmost single atom on the STM tip works as a path of current, the magnitude of current will change depending on the spatial configuration of conductor and the STM tip, obeying the superposition of sine functions reflecting confined lateral wave functions.

We note that these calculations were carried out at the temperature $T=300 \mathrm{~K}$. Since energy separation between the first and the second lateral states is comparable with $k_{B} T$, some states are relevant to the current flow. The rates of relevance of these states change gradually with the device size. In this point, the present situation is different from the point contact in which the conductance is quantized due to sudden change of the number of channel states at very low temperature [15].

We have to note that the present model for the defect is a much simplified one. In an atomistic point of view, dislocation or vacancy of atoms in reservoirs may be generated in the fabrication process. If there is a vacancy in the reservoir, many (four) bonds are disconnected. In the calculations, we found that disconnection of a bond apart from interface reduces conductivity a little. This is because, within this model, we consider that equilibrium is always satisfied in the reservoirs. We also ascertained that disconnection of a bond along $y$ direction is negligible. These results indicate that the present model that describes the defect as a disconnection of a bond at the interface along $x$-direction grasps the gist of the role of the defect. We also neglected the effect of scattering. As long as we consider atomic-scale devices, ballistic current is dominant because the time for an electron to travel from cathode to anode is shorter than characteristic time of scattering. In other words, the effect of scattering is not important. Therefore, we believe that the present effect will be realized, even though it can be modified by effect neglected in the present theory.

\section{Conflicts of Interest}

The author declares that there are no conflicts of interest regarding the publication of this paper.

\section{Acknowledgments}

The author is grateful to Mr. Y. Hanashiro for his help in developing the program code for the current calculations.

\section{References}

[1] C. Xu, H. Li, and K. Banerjee, "Modeling, analysis, and design of graphene nano-ribbon interconnects," IEEE Transactions on Electron Devices, vol. 56, no. 8, pp. 1567-1578, 2009.

[2] W. Y. Kim and K. S. Kim, "Prediction of very large values of magnetoresistance in a graphene nanoribbon device," Nature Nanotechnology, vol. 3, no. 7, pp. 408-412, 2008.

[3] H. Yan, J. Johnson, M. Law et al., "ZnO nanoribbon microcavity lasers," Advanced Materials, vol. 15, no. 22, pp. 1907-1911, 2003.

[4] G. Li, D. Liang, R. L. J. Qiu, and X. P. A. Gao, “Thermal conductivity measurement of individual $\mathrm{Bi} 2 \mathrm{Se} 3$ nano-ribbon by self-heating three- $\omega$ method," Applied Physics Letters, vol. 102, no. 4, article 043104, 2013.

[5] J. Li, Z. Qiao, X. Chen, Y. Cao, and M. He, "Gallium nitride nano-ribbon rings," Journal of Physics Condensed Matter, vol. 13, no. 14, pp. L285-L289, 2001.

[6] J. Kim, M. Lee, H. J. Shim et al., "Stretchable silicon nanoribbon electronics for skin prosthesis," Nature Communications, vol. 5, article 5747, 2014.

[7] Z. L. Zhang, Y. P. Chen, Y. E. Xie, M. Zhang, and J. X. Zhong, "Spin-polarized transport properties of Fe atomic chain adsorbed on zigzag graphene nanoribbons," Journal of Physics D: Applied Physics, vol. 44, no. 21, article 215403, 2011.

[8] A. Micheli, A. J. Daley, D. Jaksch, and P. Zoller, "Single atom transistor in a 1D optical lattice," Physical Review Letters, vol. 93, no. 14, article 140408, 2004.

[9] M. Fuechsle, J. A. Miwa, S. Mahapatra et al., "A single-atom transistor," Nature Nanotechnology, vol. 7, no. 4, pp. 242-246, 2012.

[10] C. Obermair, F. Xie, and T. Schimmel, "The single-atom transistor: Quantum electronics at room temperature," in Proceedings of the 2009 9th IEEE Conference on Nanotechnology, IEEE NANO 2009, pp. 439-441, ita, July 2009.

[11] J. Park, A. N. Pasupathy, and J. I. Goldsmith, "Coulomb blockade and the Kondo effect in single-atom transistors," Nature, vol. 417, pp. 722-725, 2002.

[12] A. J. Daley, S. R. Clark, D. Jaksch, and P. Zoller, "Numerical analysis of coherent many-body currents in a single atom transistor," Physical Review A - Atomic, Molecular, and Optical Physics, vol. 72, no. 4, article 043618, 2005.

[13] S. Datta, Quantum Transport: Atom to transistors, Cambridge University Press, 2005.

[14] M. Morifuji and Y. Maeda, "Enhancement of conductivity due to local disorder in a one-dimensional conductor," Journal of Physics Condensed Matter, vol. 23, no. 43, article 435301, 2011.

[15] B. J. Van Wees, H. Van Houten, C. W. J. Beenakker et al., "Quantized conductance of point contacts in a two-dimensional electron gas," Physical Review Letters, vol. 60, no. 9, pp. 848-850, 1988. 

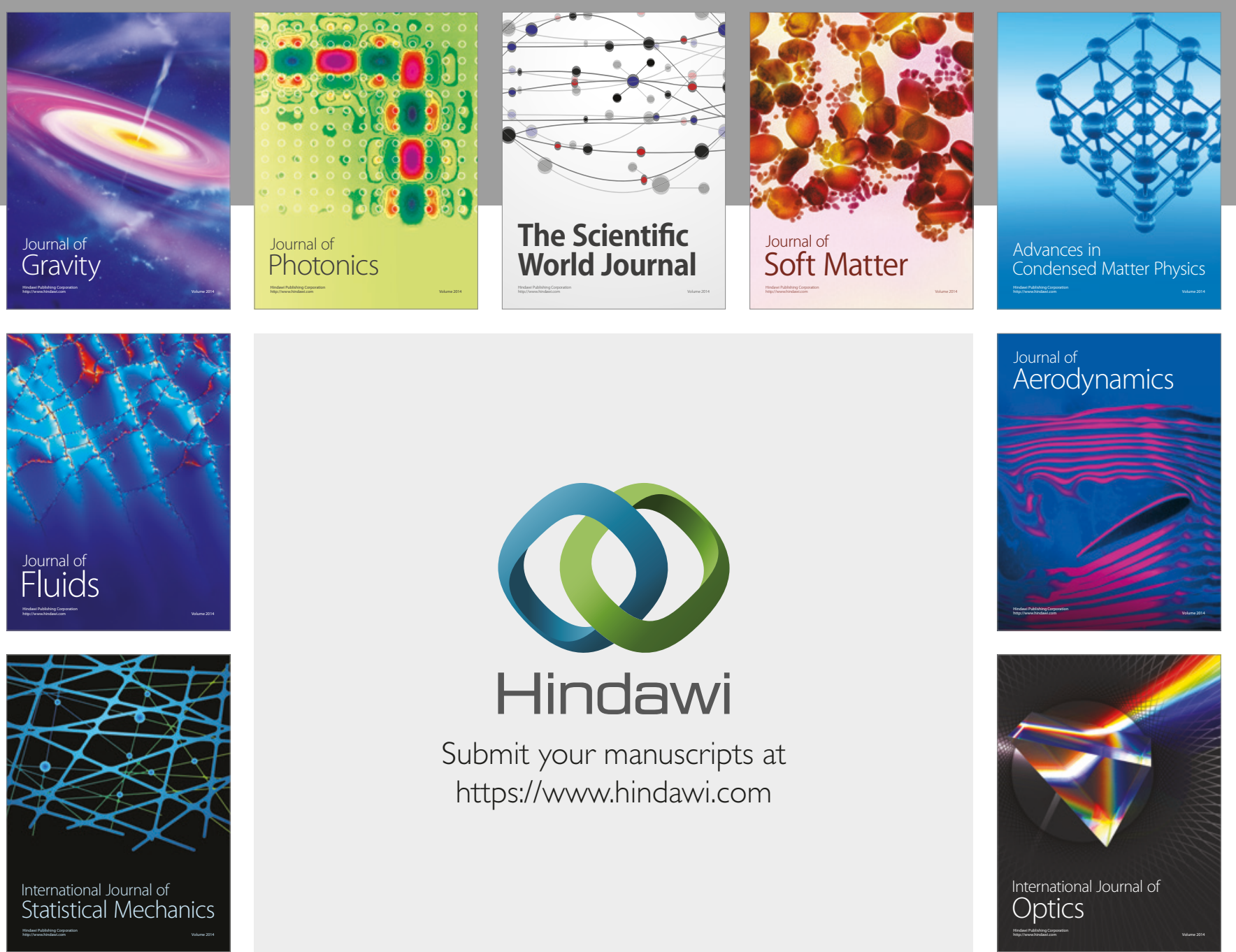

Submit your manuscripts at

https://www.hindawi.com
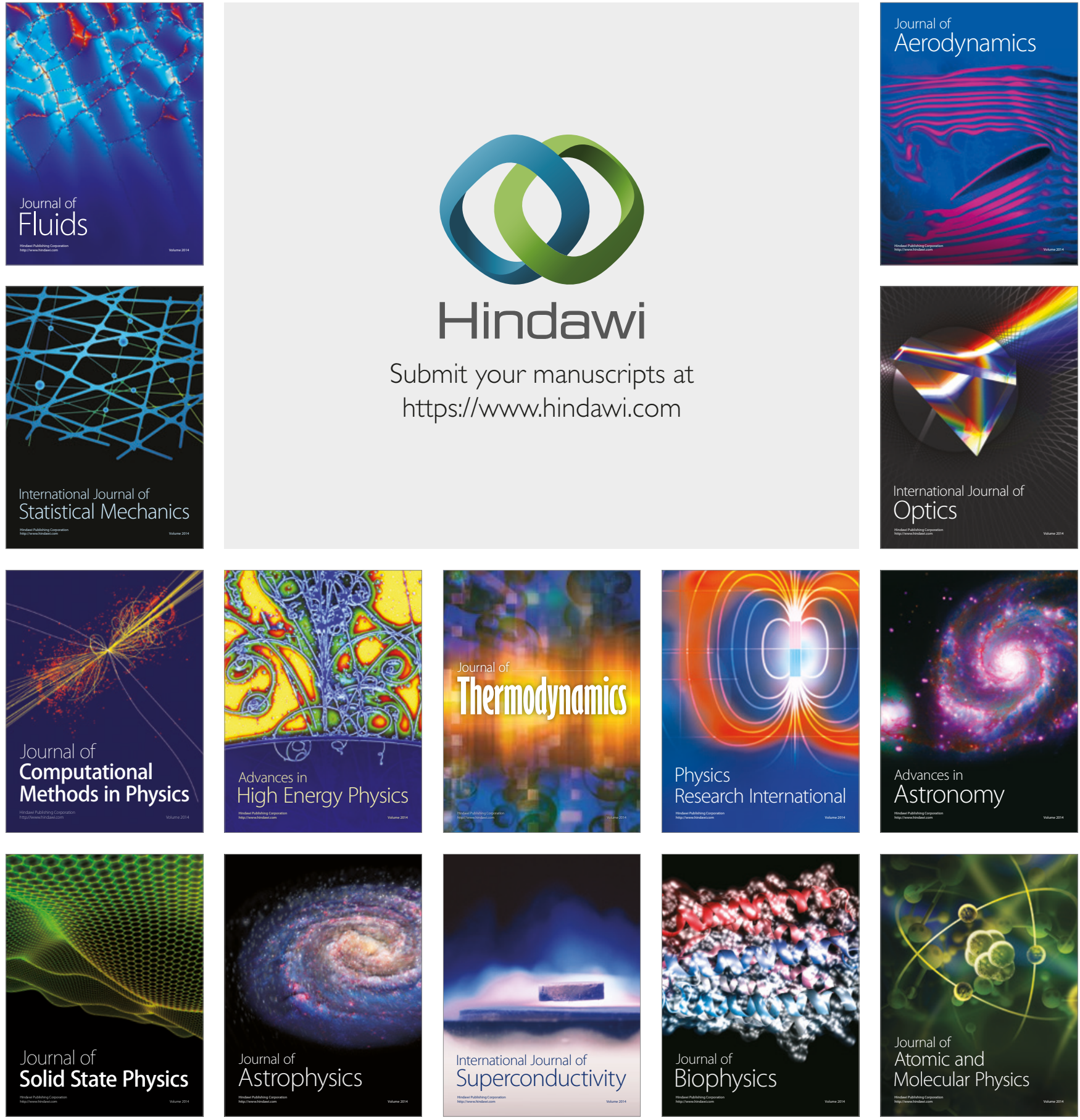